\title{
NÚMEROS ATUAIS DA CERTIFICAÇÃO FLORESTAL NO BRASIL
}

\author{
UPDATING FIGURES OF FOREST CERTIFICATION IN BRAZIL \\ Carlos Roberto Sanquetta ${ }^{1}$, Celine Mildemberg ${ }^{2}$, Leticia Maria Sella Marques Dias ${ }^{3}$ \\ 1,2,3 Universidade Federal do Paraná, Curitiba, Paraná, Brasil - sanquetta@ufpr.br, \\ mildemberg7@gmail.com \& leticiasella15@gmail.com
}

\begin{abstract}
RESUMO
A certificação florestal é um importante instrumento de gestão e garantia da sustentabilidade no setor florestal. Para avaliar o estado atual da certificação é necessário se ter números atualizados e detalhados. Este estudo visou analisar os números atuais da certificação florestal no Brasil. Para a análise, foram utilizados dados disponibilizados nas plataformas da internet dos sistemas FSC ${ }^{\circledR}$ (Forest Stewardship Council) e Cerflor/PEFC ${ }^{\circledR}$ (Sistema Brasileiro de Certificação Florestal/Plan for the Endorsement of Forest Certification Schemes), considerando os sistemas e as certificadoras, os tipos de certificação, a localização e os produtos certificados. A maior área florestal certificada no Brasil atualmente é pelo sistema FSC. Há mais florestas plantadas certificadas do que nativas. Minas Gerais é o Estado com a maior área de manejo certificada, tanto pelo FSC quanto pelo Cerflor/PEFC. Imaflora e SCS são as certificadoras com mais certificados e áreas certificadas de manejo florestal. O produto predominante declarado pelas unidades de manejo florestal certificadas é a madeira em toras. Há um número muito superior de certificações CoC (cadeia de custódia) pelo FSC do que pelo CERFLOR. A maioria das certificações CoC refere-se a indústrias de produtos madeireiros, notadamente materiais de papel e de madeira serrada. As certificadoras mais atuantes em CoC são Imaflora, SCS e Control Union. Os números da certificação florestal em manejo florestal no Brasil ainda são modestos, considerando a dimensão de sua cobertura florestal. O mesmo pode ser dito em relação ao número de indústrias certificadas em cadeia de custódia. Por isso há um amplo espaço para crescimento.
\end{abstract}

Palavras-chaves: Cadeia de custódia, Certificação Florestal, Dados setoriais, FSC, Manejo florestal, PEFC.

ABSTRACT

The forest certification is an important instrument for managing and guaranteeing sustainability in the forestry sector. To evaluate the current certification status, it is required to have updated and detailed figures. This article aimed to analyze the current numbers of forest certification in Brazil. For our analyses we used available data from the FSC (Forest Stewardship Council) and Cerflor/PEFC (Brazilian System of Forest Certification/Plan for the Endorsement of Forest Certification Schemes) online platforms, considering the most active systems and certifiers, the certification types, the location and the certified products. The largest certified forest area in Brazil is currently under the FSC program. There are more certified planted forests than certified native ones. Minas Gerais is the state with the largest certified forest management area, both by FSC and Cerflor/PEFC systems. Imaflora and SCS are the two certifiers with the greatest numbers of certification and certified forest management areas. The prevailing product, declared by the certified management forest units, is roundwood. There are much higher figures of CoC (chain of custody) certifications by FSC than by CERFLOR. Most of CoC certifications refer to wood product industries, particularly paper and sawn wood materials. The most active certifiers in CoC are Imaflora, SCS and Control Union. In conclusion, the figures of forest certification in forest management in Brazil are still modest considering the size of its forest cover. The same can be said about the numbers of certified industries in chain of custody. Therefore, there is a broad track for growth.

Keywords: Chain of custody, Forest Certification, Sector data, FSC, Forest Management, PEFC. 


\section{INTRODUÇÃO}

A sociedade atual passa por profundas transformações. De simples consumidores que compram impulsionados pelas necessidades básicas e preços, a humanidade agora adquire bens e serviços para atender outras demandas e age sob outros valores, incluindo a reflexão sobre a sustentabilidade do Planeta. As organizações precisam se posicionar adequadamente para atender às demandas da sociedade contemporânea.

Juntamente com o crescimento da produção florestal, tem surgido uma maior preocupação com os aspectos e impactos ambientais e sociais destas atividades. Todo esse movimento vem sendo impulsionado pela repercussão de notícias a respeito da destruição de florestas tropicais e os consequentes efeitos sobre o clima global (BASSO et al., 2011).

As certificações são essenciais para organizações melhorarem seus processos, produtos, serviços e se posicionarem apropriadamente, ganhando destaque nos cenários nacional e internacional. Os benefícios das certificações para as organizações extrapolam a questão financeira e mercadológica. Visam colocar as coisas em seus devidos lugares de maneira sistêmica, o que permite que a organização possa melhorar seus processos internos e oferecer produtos e serviços com mais qualidade e bem aceitos. Cada vez mais, as organizações, dos mais diversos ramos, têm exigido dos seus fornecedores certificações como forma de qualificação e garantia de atendimento aos requisitos contratuais. Isso é notável sobretudo no comércio exterior e é percebido de forma explícita no setor de base florestal (ISO 9001, 2015).

Assim, foi necessário criar mecanismos de mercado, que permitissem rotular produtos originados de florestas manejadas de forma adequada, com atividades que respeitassem os aspectos ambientais e sociais e, com isso, premiasse ou reconhecesse os produtores que adotassem este sistema de manejo. Como resposta a esta necessidade do mercado, surgiu a certificação florestal (BASSO et al., 2011).

A certificação é um procedimento voluntário que atesta um produto, processo ou serviço ao atendimento de normas e regras estabelecidas por um organismo independente da relação comercial, sendo geralmente adotada por organizações que atuam em mercados exigentes (MAYR et al., 2020). Existe um amplo espectro de certificações, entre as quais estão as de âmbito florestal que visam garantir ao consumidor que uma organização segue um conjunto específico de padrões ao bom manejo florestal e à produção florestal responsável. As normas são definidas por uma entidade, e as organizações florestais são avaliadas por auditorias externas, sendo concedido o certificado ao final do processo (ALUD, G. et al., 2008; MAYR et al., 2020).

No mundo existem dois sistemas principais de certificação: o FSC - Forest Stewardship Council (Conselho de Manejo Florestal) e o PEFC - Plan for the Endorsement of Forest Certification Schemes (Plano para 0 Reconhecimento de Esquemas de Certificação) (ALVES et al., 2011). Algumas organizações são certificadas por um ou outro sistema, mas existe um considerável número de organizações com dupla certificação (FSC, 2001).

No Brasil, a trajetória do FSC é mais antiga que a do PEFC. O FSC foi fundado em 1993 e teve seu escritório local no Brasil estabelecido em 2001. É o sistema de certificação mais aplicado no Brasil nas três modalidades: manejo florestal (FM), cadeia de custódia (CoC) e madeira controlada (CW) (FSC, 2001). O PEFC iniciou sua atuação no Brasil em cooperação com o Cerflor - Sistema Brasileiro de Certificação, a qual foi estabelecida em 2002, embora as iniciativas da certificação florestal nacional remontem o ano de 1998 (INMETRO, 2021).

Os dois principais selos de certificação florestal utilizados no Brasil, FSC e Cerflor/PEFC, têm notável aplicação no setor florestal do País, sobretudo nas plantações florestais. O FSC certificou a primeira área florestal no Brasil em 1995 e o CERFLOR foi reconhecido em 2002 pelo PEFC (SNIF, 2016; BRAGA et al., 2018).

Apesar de a certificação florestal ter se originado com a finalidade precípua de conter o desmatamento, com enfoque nas florestas nativas, no Brasil, a grande maioria das áreas certificadas são plantações florestais (BRAGA, 2018).

Existem também tipos distintos de certificação: "manejo florestal" (do inglês Forest Management - FM) que se aplica à floresta propriamente dita - sendo incluso todos os padrões: Terra Firme na Amazônia, SLIMF e Harmonizado Plantações, e a "cadeia de custódia" (CoC Chain of Custody) que se refere à rastreabilidade da matéria prima florestal dentro da indústria. Existe ainda um terceiro tipo chamado "madeira controlada", específico do sistema FSC, que visa regulamentar o uso de madeira não certificada em certificação $\mathrm{CoC}$. A certificação de manejo florestal atesta que a floresta é manejada de forma responsável (AHRENS; OLIVEIRA, 2017).

A certificação $\mathrm{CoC}$ garante a rastreabilidade da madeira e outros produtos florestais desde a produção da matéria prima das florestas até o consumidor final. A madeira controlada (CW), por sua vez, tem como objetivo orientar as empresas certificadas a evitarem produtos com origem 
florestal de categorias consideradas inaceitáveis pelo FSC, como, por exemplo, madeira originária de desmatamento ilegal (FSC, 2017; WALTER et al., 2017).

O FSC e o PEFC apresentam periodicamente os números da Certificação Florestal em seus sistemas em nível mundial. Especificamente do Brasil são também reportadas estatísticas por esses sistemas. Contudo, os números estão separados, apresentados de forma desuniforme e muito simplificada. Assim sendo, diante da necessidade de se ter informações integradas e atualizadas sobre os números da certificação florestal no Brasil, foi realizado este estudo que teve como objetivo traçar um panorama atual e detalhado do quadro das certificações florestais no País.

\section{MATERIAL E MÉTODOS}

Neste trabalho, buscou-se reunir o máximo de informações possíveis acerca de todos os aspectos relacionados à certificação FSC e Cerflor/PEFC com intuito de registrar e tomar conhecimento do andamento da certificação florestal no Brasil e no mundo. Para atingir os objetivos propostos, foi realizada, inicialmente, uma pesquisa bibliográfica, de forma online, a fim de averiguar trabalhos e publicações já existentes sobre o assunto.

Os parâmetros utilizados para a pesquisa bibliográfica foram a coleta de dados no site do PEFC (aba "Find Certified"), selecionando o tipo de certificado como "Forest Management" para Manejo Florestal e "Chain of Custody" para Cadeia de Custódia, e, para FSC, foi utilizado o site FSC Public Certificate Search, buscando por empresas brasileiras na seção "Country or Area" e, em "Certification Code", foram selecionados todos os tipos de certificação (FM, CoC, FM/CoC e CW). Para ambos, foram escolhidos apenas os certificados válidos. Além das consultas nestes bancos de dados digitais, foram utilizadas informações provenientes dos sites das certificadoras e empresas que fazem parte das organizações, as quais continham toda a base necessária para filtragem e análise delas.

Os dados foram coletados e alimentados em planilha específica, contendo as informações consideradas mais relevantes para esta pesquisa, como: tipo de organização (FSC ou Cerflor/PEFC), classificação (FM, CoC, FM/CoC), certificadora, código, status (válido ou inválido), CW, data de emissão e expiração, empresa, município, tipos de produtos, espécies vegetais utilizadas, atividades primárias e secundárias, categoria, área, unidade de manejo florestal (plantada ou nativa), tipo de empreendimento e tipo de fontes (virgens ou reciclados). Após a coleta, separação e organização dos dados em uma planilha, foi feita uma revisão e padronização das informações encontradas, fundindo as características do FSC e Cerflor/PEFC na mesma planilha. Em seguida, foi realizada análise quantitativa e respectivas interpretações resumidas neste artigo.

\section{RESULTADOS E DISCUSSÃO}

No Brasil, o sistema FSC é o que apresenta os maiores números de certificados e de áreas certificadas de Manejo Florestal (FM), totalizando 216 Unidades de Manejo Florestal (UMF) e mais de 8 milhões de hectares certificados. O sistema Cerflor/PEFC, por sua vez, apresenta 26 UMF certificadas em FM, com um total de 3,7 milhões de hectares.

Em termos de números de UFM certificadas pelo FSC destacam-se os Estados de Santa Catarina e Paraná, com 50 e 46 unidades, respectivamente. No que concerne ao Cerflor/PEFC, os Estados de São Paulo e Bahia contém o maior número de UMF certificadas, com 6 e 5 unidades certificadas, respectivamente (Tabelas 1 e 2).

Tabela 1. Número de certificados e área florestal pelo sistema FSC certificada no Brasil por Estado e tipo de floresta.

\begin{tabular}{|c|c|c|c|c|c|c|}
\hline \multirow{2}{*}{$\begin{array}{c}\text { Estad } \\
0\end{array}$} & \multicolumn{2}{|c|}{ Nativas } & \multicolumn{2}{|c|}{ Plantadas } & \multicolumn{2}{|c|}{ Total } \\
\hline & $\underset{0}{N}$ & Área (ha) & № & Área (ha) & № & Área (ha) \\
\hline MG & & & 33 & $\begin{array}{c}1.802 .54 \\
0\end{array}$ & 33 & $\begin{array}{c}1.802 .54 \\
0\end{array}$ \\
\hline MS & & & 17 & $\begin{array}{c}1.100 .13 \\
8\end{array}$ & 17 & $\begin{array}{c}1.100 .13 \\
8\end{array}$ \\
\hline SC & & & 50 & 822.165 & 50 & 822.165 \\
\hline PA & $\begin{array}{l}1 \\
0\end{array}$ & 746.853 & 3 & 72.911 & 13 & 819.764 \\
\hline SP & & & 14 & 619.122 & 14 & 619.122 \\
\hline RS & & & 15 & & 15 & 483.668 \\
\hline PR & & & 46 & 475.366 & 46 & 475.366 \\
\hline BA & & & 6 & 420.697 & 6 & 420.697 \\
\hline MA & & & 1 & 404.083 & 1 & 404.083 \\
\hline AP & 2 & 70.351 & 1 & 166.696 & 3 & 237.047 \\
\hline ES & & & 1 & 215.699 & 1 & 215.699 \\
\hline$A C$ & 1 & 190.201 & & & 1 & 190.201 \\
\hline RJ & 1 & 152.067 & & & 1 & 152.067 \\
\hline MT & & & 5 & 134.736 & 5 & 134.736 \\
\hline RO & 3 & 33.699 & 2 & 64.371 & 5 & 98.070 \\
\hline TO & & & 1 & 80.953 & 1 & 80.953 \\
\hline AM & 2 & 31.247 & & & 2 & 31.247 \\
\hline $\mathrm{GO}$ & & & 1 & 10.694 & 1 & 10.694 \\
\hline $\mathrm{AL}$ & & & 1 & 7.074 & 1 & 7.074 \\
\hline & 1 & 1.224 .41 & 19 & 6.397 .24 & 21 & 8.105 .33 \\
\hline & 9 & 8 & 7 & 4 & 6 & 1 \\
\hline
\end{tabular}

O estado de Minas Gerais é o de maior expressão em termos de áreas certificadas por FM, em ambos os 
sistemas, possuindo 1,8 milhão de hectares certificados pelo FSC e 726 mil hectares pelo sistema Cerflor/PEFC. Em segunda posição, considerando ambos os sistemas, situase o Estado de Mato Grosso do Sul, com 1,1 milhão de hectares certificados pelo FSC e 616 mil hectares pelo Cerflor/PEFC.

Tabela 2. Número de certificados e área florestal no Brasil pelo sistema Cerflor/PEFC, por Estado e tipo de floresta.

\begin{tabular}{|c|c|c|c|c|c|c|}
\hline \multirow[b]{2}{*}{ Estado } & \multicolumn{2}{|c|}{ Nativas } & \multicolumn{2}{|c|}{ Plantadas } & \multicolumn{2}{|r|}{ Total } \\
\hline & № & $\begin{array}{c}\text { Área } \\
\text { (ha) }\end{array}$ & № & & № & $\begin{array}{c}\text { Área } \\
\text { (ha) }\end{array}$ \\
\hline MG & & & 3 & 726.260 & 3 & 726.260 \\
\hline MS & & & 3 & 646.121 & 3 & 646.121 \\
\hline SP & & & 5 & 473.134 & 5 & 473.134 \\
\hline BA & & & 6 & 399.341 & 6 & 399.341 \\
\hline ES & & & 1 & 323.869 & 1 & 323.869 \\
\hline $\mathrm{MA}$ & & & 1 & 305.228 & 1 & 305.228 \\
\hline $\mathrm{RS}$ & & & 1 & 224.522 & 1 & 224.522 \\
\hline AM & 1 & 219.137 & & & 1 & 219.137 \\
\hline AP & & & 1 & 166.696 & 1 & 166.696 \\
\hline TO & & & 1 & 80.953 & 1 & 80.953 \\
\hline PA & & & 2 & 63.968 & 2 & 63.968 \\
\hline PR & & & 1 & 49.215 & 1 & 49.215 \\
\hline
\end{tabular}

Segundo Silva (1996), manejo florestal é a administração de florestas para a obtenção de benefícios econômicos e sociais, respeitando-se os mecanismos de sustentação do ecossistema. Os dados referentes às certificações em florestas nativas se tratam de florestas manejadas.

Evidenciou-se maior número de UMF certificadas em florestas plantadas em detrimento às florestas nativas, tanto para FSC quanto Cerflor/PEFC. O FSC apresenta maior número de certificados e áreas de florestas nativas certificadas em comparação com o Cerflor/PEFC. O Estado que possui maior área certificada de florestas nativas é o Pará, seguido do Acre em segundo plano, no caso do FSC. No Cerflor/PEFC a área nativa certificada pelo sistema está toda no Estado do Amazonas. Observou-se que a maciça maioria do número de certificados e áreas certificadas em florestas nativas está no bioma Amazônia.

Imaflora e SCS são as certificadoras mais bem posicionadas em termos de números de certificados concedidos e área certificada de FM, considerando ambos os sistemas de certificação. Essas duas certificadoras detêm quase $90 \%$ do mercado em termos de número de certificados e áreas florestais certificadas no Brasil. Bureau Veritas encontra-se em posição intermediária e Control
Union e Rina têm atuações em menor escala. Particularizando para as florestas nativas, observa-se que o Imaflora é a certificadora que tem maior atuação, haja vista que são poucos os casos certificados pela SCS nessa tipologia florestal (Tabela 3).

Tabela 3. Número de certificados e área florestal certificada no Brasil por sistema de certificação, tipo de floresta e certificadora.

\begin{tabular}{|c|c|c|c|c|c|c|}
\hline \multirow{2}{*}{$\begin{array}{c}\text { Certificador } \\
\text { a }\end{array}$} & \multicolumn{2}{|c|}{ Nativas } & \multicolumn{2}{|c|}{ Plantadas } & \multicolumn{2}{|r|}{ Total } \\
\hline & $\begin{array}{l}\mathbf{N} \\
\underline{0}\end{array}$ & $\begin{array}{l}\text { Área } \\
\text { (ha) }\end{array}$ & № & Área (ha) & № & Área (ha) \\
\hline \multicolumn{7}{|c|}{ Sistema FSC } \\
\hline SCS & 3 & 20.253 & 96 & $\begin{array}{c}3.763 .49 \\
4\end{array}$ & 99 & $\begin{array}{c}3.783 .74 \\
7\end{array}$ \\
\hline IMAFLORA & $\begin{array}{l}1 \\
6\end{array}$ & $\begin{array}{c}929.17 \\
9\end{array}$ & 77 & $\begin{array}{c}2.846 .21 \\
0\end{array}$ & 93 & $\begin{array}{c}3.775 .38 \\
9\end{array}$ \\
\hline $\begin{array}{l}\text { BUREAU } \\
\text { VERITAS }\end{array}$ & & & 18 & 525.817 & 18 & 525.817 \\
\hline $\begin{array}{l}\text { CONTROL } \\
\text { UNION }\end{array}$ & & & 6 & 20.377 & 6 & 20.377 \\
\hline TOTAL & $\begin{array}{l}1 \\
9\end{array}$ & $\begin{array}{c}949.43 \\
2\end{array}$ & $\begin{array}{c}19 \\
7\end{array}$ & $\begin{array}{c}7.155 .89 \\
8\end{array}$ & $\begin{array}{c}21 \\
6\end{array}$ & $\begin{array}{c}8.105 .33 \\
1\end{array}$ \\
\hline \multicolumn{7}{|c|}{ Sistema Cerflor/PEFC } \\
\hline IMAFLORA & 1 & $\begin{array}{c}219.13 \\
7\end{array}$ & 11 & $\begin{array}{c}1.540 .45 \\
3\end{array}$ & 12 & $\begin{array}{c}1.759 .59 \\
0\end{array}$ \\
\hline SCS & & & 10 & $\begin{array}{c}1.283 .60 \\
7\end{array}$ & 10 & $\begin{array}{c}1.283 .60 \\
7\end{array}$ \\
\hline $\begin{array}{l}\text { BUERAU } \\
\text { VERITAS }\end{array}$ & & & 3 & 404.629 & 3 & 404.629 \\
\hline RINA & & & 1 & 230.617 & 1 & 230.617 \\
\hline Total & 1 & $\begin{array}{c}219.13 \\
7\end{array}$ & 25 & $\begin{array}{c}3.459 .30 \\
6 \\
\end{array}$ & 26 & $\begin{array}{c}3.678 .44 \\
3 \\
\end{array}$ \\
\hline
\end{tabular}

No que diz respeito aos produtos declarados nas florestas certificadas, a grande maioria refere-se à madeira em toras, em ambos os sistemas. Outros produtos madeireiros são também declarados, em menor número, mas superam os produtos florestais não madeireiros, como os alimentos, gomas, óleos, entre outros. Algumas UMF declaram mais que um produto, motivo pelo qual as das áreas reportadas na totalização dos produtos não coincidem com as áreas efetivamente certificadas (Tabela 4).

No que tange à cadeia de custódia e à madeira controlada, destacam-se os produtos de papelaria, impressos, papel de embalagem, produtos de papel em geral, madeira serrada e beneficiada, madeira para construção, móveis de interior e painéis de madeira. A maioria dos certificados CoC são pelo sistema FSC, sendo o número de certificados $\mathrm{CoC}$ no sistema Cerflor/PEFC bastante diminuto. Os certificados CW (madeira controlada) são exclusivos do FSC e centram-se nos seguintes produtos: papel, madeira em tora, madeira para cavacos e partículas, madeira serrada e beneficiada e madeira para construção. A certificação de cadeia de 
custódia tem sido utilizada quase que exclusivamente na

industrialização de produtos madeireiros (Tabela 5).

Tabela 4. Número de certificados e área florestal certificada no Brasil por sistema e tipo de produto declarado.

\begin{tabular}{|c|c|c|c|c|c|c|}
\hline \multirow{2}{*}{ Tipo de Produto } & \multicolumn{2}{|c|}{ NATIVAS } & \multicolumn{2}{|c|}{ PLANTADAS } & \multicolumn{2}{|c|}{ TOTAL } \\
\hline & № & Área (ha) & № & Área (ha) & № & Área (ha) \\
\hline \multicolumn{7}{|c|}{ Sistema FSC } \\
\hline Madeira em tora & 17 & 798.467 & 194 & 7.129 .488 & 211 & 7.927 .955 \\
\hline Madeira para cavaco ou partículas & 1 & 33.554 & 18 & 244.173 & 19 & 277.727 \\
\hline Alimento & 2 & 150.965 & 2 & 19.260 & 4 & 170.225 \\
\hline Madeira serrada e beneficiada & & & 7 & 143.357 & 7 & 143.357 \\
\hline Gomas, óleos e derivados & 1 & 91.556 & 3 & 31.064 & 4 & 122.621 \\
\hline Outros produtos não madeireiros & 1 & 91.556 & & & 1 & 91.556 \\
\hline Casca & 1 & 7.074 & 1 & 75.176 & 2 & 82.250 \\
\hline Madeira para carvão & & & 1 & 75.176 & 1 & 75.176 \\
\hline Plantas ou suas partes & 1 & 33.554 & 3 & 37.320 & 4 & 70.874 \\
\hline Madeira tratada e impregnada & & & 5 & 51.523 & 5 & 51.523 \\
\hline \multicolumn{7}{|l|}{ Polpa } \\
\hline Total & 24 & 1.206 .728 & 234 & 7.806 .538 & 258 & 9.013.266 \\
\hline \multicolumn{7}{|c|}{ Sistema Cerflor/PEFC } \\
\hline Madeira em tora & 1 & 219.137 & 22 & 3.014 .524 & 23 & 3.233 .661 \\
\hline Madeira para cavaco ou partículas & & & 2 & 359.719 & 2 & 359.719 \\
\hline Madeira para carvão & & & 1 & 230.617 & 1 & 230.617 \\
\hline Madeira serrada e beneficiada & 1 & 219.137 & & & 1 & 219.137 \\
\hline Polpa & & & 2 & 214.165 & 2 & 214.165 \\
\hline \multicolumn{7}{|l|}{ Casca } \\
\hline \multicolumn{7}{|l|}{ Plantas ou suas partes } \\
\hline \multicolumn{7}{|l|}{ Gomas, óleos e derivados } \\
\hline \multicolumn{7}{|l|}{ Alimento } \\
\hline \multicolumn{7}{|l|}{ Outros produtos não madeireiros } \\
\hline \multicolumn{7}{|l|}{ Madeira tratada e impregnada } \\
\hline TOTAL & 2 & 438.274 & 27 & 3.819 .025 & 29 & 4.257.300 \\
\hline
\end{tabular}

Três certificadoras são responsáveis por mais de $75 \%$ das certificações CoC pelo FSC, isto é, Imaflora, SCS e Control Union, com $26,04 \%$, 23,38\% e $22,66 \%$ dos casos, respectivamente. O Imaflora é responsável por mais de dois terços das certificações CW pelo FSC, seguido da SCS, com 18,31\%. Há certo equilíbrio na atuação das certificadoras em $\mathrm{CoC}$ pelo sistema Cerflor/PEFC.

No mundo, o Programme for the Endorsement of Forest Certification (PEFC) é o sistema com maior área certificada de Manejo Florestal ou Forest Management (FM), com 325 milhões de hectares certificados e mais de 20 mil certificados CoC. O Forest Stewardship Council (FSC) é o sistema de certificação florestal que detém o maior número de certificados de Cadeia de Custódia ou Chain of Custody (CoC) no mundo, com mais de 43 mil certificados e 211 milhões de hectares (CALIXTO, 2020).

Neste estudo, isso ficou muito evidenciado, pois $79 \%$ das áreas certificadas pelo FSC e $94 \%$ pelo Cerflor/PEFC são de florestas plantadas. Isso está atrelado à performance mais arrojada desse segmento, que atualmente é responsável por mais de $86 \%$ da produção florestal do país em volume de madeira (SFB, 2019).

Apesar da área relativamente diminuta das florestas plantadas em comparação com as florestas nativas, é nelas que reside a maior pujança da economia florestal do País. Diante disso, pode-se dizer que o segmento de florestas plantadas efetivamente abraçou a certificação florestal como instrumento de gestão. Considerando que a área plantada no Brasil é de cerca de 10 milhões de hectares (SANQUETTA et al., 2018a), deduz-se que mais de $68 \%$ das florestas estão certificadas em FM pelo FSC e $35 \%$ pelo Cerflor/PEFC. Considerando a área de 486 milhões de hectares, menos de $0,25 \%$ das florestas nativas brasileiras estão certificadas por um ou outro sistema, considerando apenas mata nativa manejada com exploração (SANQUETTA et al., 2018b). Conforme os dados registrados na tabela 3, o total da área certificada de florestas plantadas é de 10.615.204 hectares, enquanto a área de florestas nativas é de 1.168 .569 hectares. Spathelf et al. (2004) questionam o poder da certificação florestal na 
Tabela 5. Número de certificados de cadeia de custódia e madeira controlada no Brasil, por sistema e tipo de produto declarado.

\begin{tabular}{|c|c|c|c|}
\hline \multirow{2}{*}{ Tipo de Produto } & \multicolumn{2}{|c|}{ Sistema FSC } & \multirow{2}{*}{$\begin{array}{c}\text { Sistema Cerflor/PEFC } \\
\text { CoC }\end{array}$} \\
\hline & $\mathrm{CoC}$ & CW & \\
\hline Produtos de papelaria & 443 & - & 2 \\
\hline Materiais impressos & 443 & - & 3 \\
\hline Papel de embalagem & 407 & 9 & 8 \\
\hline Papel & 277 & 67 & 8 \\
\hline Outros produtos de papel e polpa & 269 & 20 & 13 \\
\hline Madeira serrada e beneficiada & 246 & 54 & - \\
\hline Madeira para construção & 201 & 56 & 1 \\
\hline Móveis de interior & 200 & 29 & 4 \\
\hline Painéis de madeira & 191 & 41 & 1 \\
\hline Madeira em tora & 187 & 66 & - \\
\hline Madeira para cavacos e partículas & 173 & 67 & 1 \\
\hline Produtos aplainados & 160 & 51 & - \\
\hline Papel cartão & 143 & 15 & - \\
\hline Papeis sanitários e domésticos & 97 & 17 & 1 \\
\hline Madeira engenheirada & 96 & 39 & - \\
\hline Papel e cartão corrugado & 95 & 13 & 1 \\
\hline Móveis de exterior e jardinagem & 83 & 5 & 2 \\
\hline Lâminas & 77 & 17 & - \\
\hline Polpa & 76 & 30 & 6 \\
\hline Produtos domésticos & 61 & 25 & - \\
\hline Madeira para embalagem e similares & 37 & 13 & - \\
\hline Bobinas, carretéis, rolos, etc. & 36 & 1 & - \\
\hline Outros produtos madeireiros & 33 & 1 & 4 \\
\hline Madeira tratada e impregnada & 29 & 3 & - \\
\hline Outras manufaturas de madeira & 28 & - & - \\
\hline Venda de artefatos de madeira & 17 & - & - \\
\hline Produtos de recreação & 16 & - & - \\
\hline Gomas, óleos e derivados & 12 & 8 & - \\
\hline Casca & 9 & 1 & - \\
\hline Madeira para carvão & 9 & - & - \\
\hline Instrumentos musicais & 8 & - & - \\
\hline Químicos, medicinais e derivados & 4 & - & - \\
\hline Condicionadores do solo e substratos & 2 & - & - \\
\hline Cortiça e artigos de cortiça & 2 & - & - \\
\hline Bambu e artigos de bambu & 2 & - & - \\
\hline Alimento & 2 & - & 1 \\
\hline Outros produtos não madeireiros & 1 & - & - \\
\hline Plantas e suas partes & 0 & - & - \\
\hline Total & 4.172 & 648 & 56 \\
\hline
\end{tabular}

De forma geral, as áreas de florestas plantadas certificadas seguem proporcionalmente a sua distribuição nos Estados da Federação. Os Estados que detêm mais áreas de florestas plantadas são aqueles que possuem maiores extensões de florestas certificadas (tabela 1 e 2). Tal lógica não se aplica bem às florestas nativas.

Alves et al. (2009a) apontam que a maior parte das empresas pesquisadas no setor moveleiro (67\%) detêm certificação florestal como estratégia para facilitar as suas exportações. Como a maior parte das exportações de produtos florestais brasileiros são madeireiros, e como a produção de não madeireiros é menos expressiva do ponto de vista econômico-financeiro (SNIF, 2016), era de se esperar maior motivação para certificar florestas destinadas à industrialização de madeira. É o que se verifica inequivocamente no presente estudo, que 
demonstra a predominância de produtos elaborados a partir de madeira roliça certificada.

Fischer \& Waquil (2009) analisaram a influência da certificação florestal no desempenho exportador do setor de celulose e papel do Brasil. Concluíram parcialmente que o uso crescente de certificações não interfere positivamente sobre o desempenho do comércio exterior da indústria brasileira de papel e celulose Já Paiva et al. (2014), ao estudarem o caso de uma grande empresa produtora de celulose e papel, apontam que a certificação florestal é instrumento de diferenciação no mercado e atualmente se torna fundamental no segmento de celulose e papel, principalmente para empresas motivadas pela exportação.

Em estudo recente, Mayr et al. (2020) analisaram se a certificação florestal traz benefícios significativos para as empresas brasileiras. Os autores concluíram que existem reais benefícios para as empresas certificadas, mas que os alcances pretendidos não são totalmente atingidos, sendo necessários aprimoramentos.

\section{CONCLUSÕES}

A partir dos resultados e discussões apresentados neste trabalho, conclui-se que:

- A maioria das florestas plantadas estabelecidas no País já está certificada, ao passo que uma fração mínima das florestas nativas se encontra em tal situação;

- O sistema de certificação mais utilizado no Brasil é o FSC, mas seu concorrente, o sistema Cerflor/PEFC, apresenta números expressivos;

- Imaflora e SCS são as certificadoras mais atuantes em certificação de manejo florestal e cadeia de custódia;

- Os segmentos de materiais produzidos a partir do papel, de madeira sólida e de painéis de madeira são os mais ativos na certificação cadeia de custódia;

- Há espaço para crescimento da certificação florestal no País, pois o número de certificados e a área florestal certificada em manejo florestal para nativas ainda são pequenos, considerando a cobertura florestal do País;

- Há espaço para crescimento da certificação CoC, dado o grande número de indústrias ainda não certificadas pela cadeia de custódia no País que, segundo Alves, et al. (2009b), é de 33\%.

\section{AGRADECIMENTOS}

O primeiro autor agradece ao CNPq pela bolsa de produtividade em pesquisa - PQ (Processo 304013/2018-8) e ao apoio em projeto de pesquisa (Processo 438875/2018-
4).

A segunda autora agradece à Universidade Federal do Paraná, ao professor Carlos Roberto Sanquetta e ao CNPq pela oportunidade de pesquisa.

\section{REFERÊNCIAS}

AHRENS, S.; OLIVEIRA, Y.M.M. Plantações florestais comerciais, a certificação e os diálogos setoriais. Brasília: Embrapa, 2017.

AULD, G. et al. Certification schemes and the impacts on forests and forestry. Annual Review of Environment and Resources, v.33, p.187-211, 2008

ALVES, R.R. et al. Certificação Florestal e o consumidor final: um estudo no polo moveleiro de Ubá, MG. Floresta e Ambiente, v.16, n.2, p.40-48, 2009a.

ALVES, R.R. et al. Certificação florestal e o mercado moveleiro nacional. Árvore, v.33, n.3, p.583-589, 2009b.

ALVES, R.R. et al. Certificação florestal na visão gerencial e estratégica do polo moveleiro de Ubá, MG. Cerne, v.13, n.1, p.117-122, 2007.

BASSO, V.M. et al. Certificação florestal em grupo no Brasil. Floresta e Ambiente, v.18, n.2, p.160-170, 2011.

BRAGA, W.R.O. et al. Certificação florestal - acesso a mercado ou mercado de acesso? DRd - Desenvolvimento Regional em Debate, v.8, n.1, p.182-197, 2018.

CALIXTO, G.A.S. A certificação como processo de melhoria da sustentabilidade dos sistemas florestais. Bragança: Instituto Politécnico de Bragança, 2020.

FISCHER, B.B.; WAQUIL, P.D. Sistemas de certificação florestal no setor brasileiro de papel e celulose: influências no desempenho exportador. São Paulo: Instituto de Economia Agrícola, 2009.

SFB. Florestas do Brasil em resumo. Brasília, 2019. Disponível em: <https://www.florestal.gov.br/publicacoes/1737-florestas-dobrasil-em-resumo-2019>.

FSC. FSC Brasil. 2021.Disponível em: https://br.fsc.org/pt-br.

FSC. Fatos e números no brasil e no mundo. 2021. Disponível em: https://br.fsc.org/pt-br/fsc-brasil/fatos-e-nmeros.

FSC. Public Certificate Search. 2021. Disponível em: https://info.fsc.org/certificate.php.

INMETRO. Cerflor: Certificação florestal. 2021. Disponível em: https://www.gov.br/inmetro/pt-br.

MAYR, G.G.O. et al. A certificação florestal traz benefícios para as empresas brasileiras? Brazilian Journal of Development, v.6, n.6, p.39291-39303, 2020.

PAIVA, S. et al. A certificação florestal pelo FSC: um estudo de caso. Revista Floresta, v.45, n.2, p.213-222, 2014. 
PEFC - Programme for the Endorsement of Forest Certification. Find Certified. 2021. Disponivel em: https://www.pefc.org/findcertified/advanced

SANQUETTA, C.R. et al. Dinâmica em superfície, volume, biomassa e carbono nas florestas plantadas brasileiras: 19902016. BIOFIX Scientific Journal, v.3, n.1, p.152-160, 2018a.

SANQUETTA, C.R. et al. Dinâmica em superfície, volume, biomassa e carbono nas florestas nativas brasileiras: 1990-2015. BIOFIX Scientific Journal, v.3, n.1, p.193-198, 2018b.

SILVA, J.N.M. Manejo florestal. Brasília: EMBRAPA-SPI; Belém: EMBRAPA-CPATU, 1996.

SNIF. Produção florestal. 2016 Disponível em: https://www.florestal.gov.br/publicacoes/818-boletim-snif2016-producao-florestal

SPATHELF, P. et al. Certificação florestal no brasil - uma ferramenta eficaz para a conservação das florestas naturais? Revista Floresta, v.34, p.373-379, 2004.

WALTER, T.S. et. al. Quantificação e distribuição espacial dos certificados florestais FSC no Brasil. Agrarian Academy, v.4, n.8, p.228-239, 2017.

Recebido em 17-05-2021

Aceito em 16-07-2021 